\title{
Spontaneous polarization and piezoelectricity in boron nitride nanotubes
}

\author{
S. M. Nakhmanson, ${ }^{1, *}$ A. Calzolari, ${ }^{2}$ V. Meunier, ${ }^{1,3}$ J. Bernholc, ${ }^{1,3}$ and M. Buongiorno Nardelli ${ }^{1,3, \uparrow}$ \\ ${ }^{1}$ Department of Physics, North Carolina State University, Raleigh, North Carolina 27695, USA \\ ${ }^{2}$ INFM, National Research Center on nanoStructures and bioSystems at Surfaces (S3) and Dipartimento di Fisica, \\ Università di Modena e Reggio Emilia, Modena, Italy \\ ${ }^{3}$ Center for Computational Sciences (CCS) and Computer Science and Mathematics Division, Oak Ridge National Laboratory, \\ Oak Ridge, Tennesse 37830, USA
}

(Received 21 February 2003; published 10 June 2003)

\begin{abstract}
$A b$ initio calculations of the spontaneous polarization and piezoelectric properties of boron nitride nanotubes show that they are excellent piezoelectric systems with response values larger than those of piezoelectric polymers. The intrinsic chiral symmetry of the nanotubes induces an exact cancellation of the total spontaneous polarization in ideal, isolated nanotubes of arbitrary indices. Breaking of this symmetry by intertube interaction or elastic deformations induces spontaneous polarization comparable to those of wurtzite semiconductors.
\end{abstract}

DOI: 10.1103/PhysRevB.67.235406

PACS number(s): 73.22. $-\mathrm{f}, 77.65 .-\mathrm{j}, 77.70 .+\mathrm{a}, 85.35 . \mathrm{Kt}$

\section{INTRODUCTION}

Piezoelectric and pyroelectric materials for modern technological applications should display an excellent piezoelectric response, combined with high mechanical stability and low environmental impact. Existing materials, which can be broadly divided into the families of ceramics and polymers, can only partially fulfill the above requirements. Lead zirconate titanate (PZT) ceramics, for example, are strong piezoelectrics and pyroelectrics ${ }^{1,2}$ but, unfortunately, they are also brittle, heavy, and toxic. On the other hand, polymers such as polyvinylidene fluoride (PVDF) are lightweight, flexible, and virtually inert, but their polar properties are an order of magnitude weaker than those of PZT. ${ }^{3}$ In this paper, we examine spontaneous polarization and piezoelectricity in boron nitride nanotubes (BNNT's) in order to estimate their potential usefulness in various pyroelectric and piezoelectric device applications, and to understand the interplay between symmetry and polarization in nanotubular systems.

BNNT's, broadly investigated since their initial prediction $^{4}$ and succeeding discovery, ${ }^{5}$ are already well known for their excellent mechanical properties. ${ }^{6}$ However, unlike carbon nanotubes (CNT's), most of BN structures are noncentrosymmetric and polar, which might suggest the existence of nonzero spontaneous polarization fields. Recently, these properties have been partially explored by Mele and Král, using a model electronic Hamiltonian. ${ }^{7}$ They predicted that BNNT's are piezoelectric and pyroelectric, with the direction of the spontaneous electric field that changes with the index of the tubes. The $a b$ initio calculations presented in this paper provide a much fuller description and show that BNNT systems are indeed excellent lightweight piezoelectrics, with comparable or better piezoelectric response and superior mechanical properties than in piezoelectric polymers. However, contrary to the conclusions of Ref. 7, our combined Berry phase and Wannier function (WF) analysis demonstrates that electronic polarization in BNNT's does not change its direction but rather grows monotonically with the increasing diameter of the tube. Furthermore, the electronic and ionic spontaneous polarizations in BNNT's cancel exactly and these systems are pyroelectric only if their intrinsic helical symmetry is broken by, e.g., intertube interactions or elastic distortions.

The rest of this paper is organized as follows: Sec. II briefly reviews the formulation of the modern polarization theory in terms of Berry phases or Wannier functions. It also presents the details of the numerical techniques that were used to compute polarization. In Sec. III we discuss the results and the complementary nature of the two techniques to compute the spontaneous polarization. Finally, Sec. IV presents the summary and conclusions.

\section{COMPUTATIONAL METHODS}

\section{A. Modern theory of polarization}

The problem of computing polarization in materials is very subtle and is best approached by the "Berry-phase" method, introduced only a decade ago by Vanderbilt and King-Smith, ${ }^{8}$ and Resta. ${ }^{9}$ Within this approach, the polarization difference between two states of a system is computed as a geometrical quantum phase. In practice, this difference, $\Delta \boldsymbol{P}=\boldsymbol{P}^{\left(\lambda_{1}\right)}-\boldsymbol{P}^{\left(\lambda_{0}\right)}$, can be obtained if one can find an adiabatic transformation $\lambda$ from one state to the other that leaves the system insulating. In the spirit of Ref. $10, \boldsymbol{P}^{(\lambda)}$ can be split into two parts: $\boldsymbol{P}_{i o n}^{(\lambda)}$ and $\boldsymbol{P}_{e l}^{(\lambda)}$, corresponding to the ionic and electronic contributions respectively. In the case of paired electron spins, the expression for the total polarization of the system can be written as follows:

$$
\begin{aligned}
\boldsymbol{P}^{(\lambda)}= & \boldsymbol{P}_{i o n}^{(\lambda)}+\boldsymbol{P}_{e l}^{(\lambda)}=\frac{e}{V} \sum_{\tau} Z_{\tau}^{(\lambda)} \boldsymbol{r}_{\tau}^{(\lambda)} \\
& -\frac{2 i e}{8 \pi^{3}} \sum_{i} \int_{B Z} d \boldsymbol{k}\left\langle u_{i \boldsymbol{k}}^{(\lambda)}\left|\nabla_{k}\right| u_{i \boldsymbol{k}}^{(\lambda)}\right\rangle,
\end{aligned}
$$

where $V$ is the volume of the unit cell, $Z_{\tau}$ and $\boldsymbol{r}_{\tau}$ are the charge and position of the $\tau$ th atom in the cell, and $u_{i k}$ are the occupied cell-periodic Bloch states of the system. For the electronic part, an electronic phase $\varphi_{\alpha}^{(\lambda)}$ (Berry phase) defined modulo $2 \pi$ can be introduced as 


$$
\varphi_{\alpha}^{(\lambda)}=V \boldsymbol{G}_{\alpha} \cdot \boldsymbol{P}_{e l}^{(\lambda)} / e,
$$

where $\boldsymbol{G}_{\alpha}$ is the reciprocal lattice vector in the $\alpha$ direction. Similarly, one can construct an angular variable for the ionic part, called, in what follows, the "ionic" phase, so that the total geometrical phase is

$$
\Phi_{\alpha}^{(\lambda)}=\sum_{\tau} Z_{\tau}^{(\lambda)} \boldsymbol{G}_{\alpha} \cdot \boldsymbol{r}_{\tau}^{(\lambda)}+\varphi_{\alpha}^{(\lambda)} .
$$

The total polarization in the $\alpha$ direction becomes

$$
\boldsymbol{P}_{\alpha}^{(\lambda)}=e \Phi_{\alpha}^{(\lambda)} \boldsymbol{R}_{\alpha} / V
$$

where $\boldsymbol{R}_{\alpha}$ is the real-space lattice vector corresponding to $\boldsymbol{G}_{\alpha},\left(\boldsymbol{R}_{\alpha} \cdot \boldsymbol{G}_{\alpha}\right)=1$.

Alternatively, the electronic polarization of a system can be expressed in terms of the centers of charge of the Wannier functions of its occupied bands: 8,9

$$
\boldsymbol{P}_{e l}^{(\lambda)}=-\frac{2 e}{V} \sum_{i} \int \boldsymbol{r}\left|W_{i}^{(\lambda)}(\boldsymbol{r})\right|^{2} d \boldsymbol{r}=-\frac{2 e}{V} \sum_{i}\left\langle\boldsymbol{r}_{i}^{(\lambda)}\right\rangle,
$$

where the WF $W_{i}^{(\lambda)}(\boldsymbol{r})$ is constructed from the Bloch eigenstates $u_{i k}^{(\lambda)}$ of band $i$ using the unitary transformation

$$
W_{i}(\boldsymbol{r})=\frac{V}{(2 \pi)^{3}} \int_{B Z} e^{i k r} u_{i k}(\boldsymbol{r}) d \boldsymbol{k},
$$

and $\left\langle\boldsymbol{r}_{i}^{(\lambda)}\right\rangle$ is the center of charge for the $\mathrm{WF} W_{i}^{(\lambda)}$. However, because of the arbitrariness in the choice of the phases of the Bloch orbitals [nonuniqueness of transformation (6)], there is no unique representation of the WF's of a given group of bands. In our approach, we employ an algorithm that has been recently developed by Marzari and Vanderbilt, ${ }^{11}$ which exploits the freedom in transformation (6) to construct WF's that are as localized as possible. This is achieved by minimizing the sum of the quadratic spreads of the Wannier probability distributions $\left|W_{j}(\boldsymbol{r})\right|^{2}$,

$$
\Omega=\sum_{j=1}^{M}\left[\left\langle r_{j}^{2}\right\rangle-\left\langle\mathbf{r}_{j}\right\rangle^{2}\right],
$$

where the sum is over an isolated group of bands. The maximally localized WF's generated by this procedure are real, apart from an overall phase factor.

In the both methods presented above, $\boldsymbol{P}_{e l}^{(\lambda)}$ can be obtained only modulo $2 e \boldsymbol{R} / V$ due to the arbitrariness in the choice of the phases of the Bloch functions. However, the difference in polarization $\Delta \boldsymbol{P}$ is well defined if $\left|\Delta \boldsymbol{P}_{e l}\right| \ll|2 e \boldsymbol{R} / V|$. The same indetermination issues apply to $\boldsymbol{P}_{\text {ion }}^{(\lambda)} \cdot{ }^{10}$

\section{B. Calculations}

In computing the spontaneous polarization as the difference between a polar BNNT and a nonpolar reference state, a natural choice for the nonpolar state is a nanotube of the same geometry, but with boron and nitrogen atoms substituted by "pseudocarbon" atoms, which are $50 \%$ boron and $50 \%$ nitrogen. The adiabatic transformation is then defined by a "virtual crystal" procedure, in which parameter $\lambda$ cor-

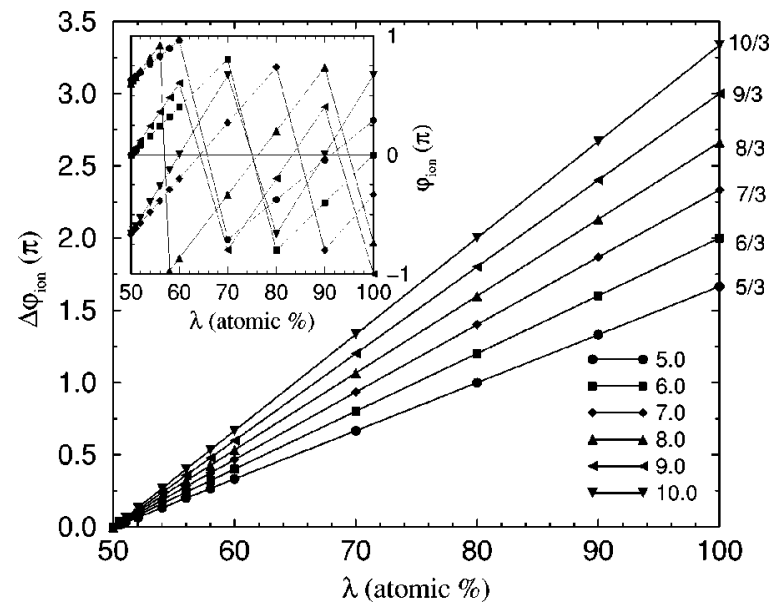

FIG. 1. Ionic-phase difference between the polar and nonpolar configurations for zigzag nanotubes; the ionic phase of the nonpolar configuration is set to zero. Inset: ionic phases wrapped into the $[-\pi, \pi]$ interval. Phases are given in units of $\pi$.

responds to the content (in at\%) of a site that is transformed from pure boron to the nonpolar reference state (vice versa for nitrogen sites).

We used an ab initio multigrid-based total-energy method, employing a real-space grid as a basis, ${ }^{12}$ for all the Berryphase calculations presented here. The Ceperley-Alder ${ }^{13}$ form, parametrized by Perdew and Zunger, ${ }^{14}$ was used for the exchange-correlation energy functional in the localdensity approximation. The norm-conserving pseudopotentials ${ }^{15}$ for all the elements, including "virtual" ones, were generated by the FHI98PP package ${ }^{16}$ utilizing the Kleinman-Bylander formulation. ${ }^{17}$

To isolate the contribution of individual nanotubes, we performed polarization calculations for periodic crystals of noninteracting (i.e., positioned sufficiently far apart) nanotubes in hexagonal and tetragonal arrangements. The electronic structure calculations were carried out using two special $k$ points along the $\Gamma-A$ direction in the hexagonal or $\Gamma-Z$ direction in the tetragonal Brillouin zones. The $k$-space integration to compute $\varphi_{z}^{(\lambda)}$ was done on a string of $20 k$ points uniformly distributed along the same direction and shifted to avoid the $\Gamma$ point. The internal consistency of our approach was checked against the results obtained using the ABINIT code $^{18}$ for a few selected systems with excellent agreement.

Because of the different alignments of the polar bond with respect to the nanotube axis, we anticipate that the symmetry of the nanotube will play an important role in determining the magnitude of the spontaneous polarization field. In particular, since the zigzag geometry maximizes the axial dipole moment, we expect to observe the strongest effects in $(n, 0)$ nanotubes.

\section{RESULTS AND DISCUSSION}

\section{A. Berry-phase method}

The ionic part of the polarization in zigzag BNNT's, presented in Fig. 1, is large and directly proportional to the nanotube's index. This is in contrast, for instance, to the 


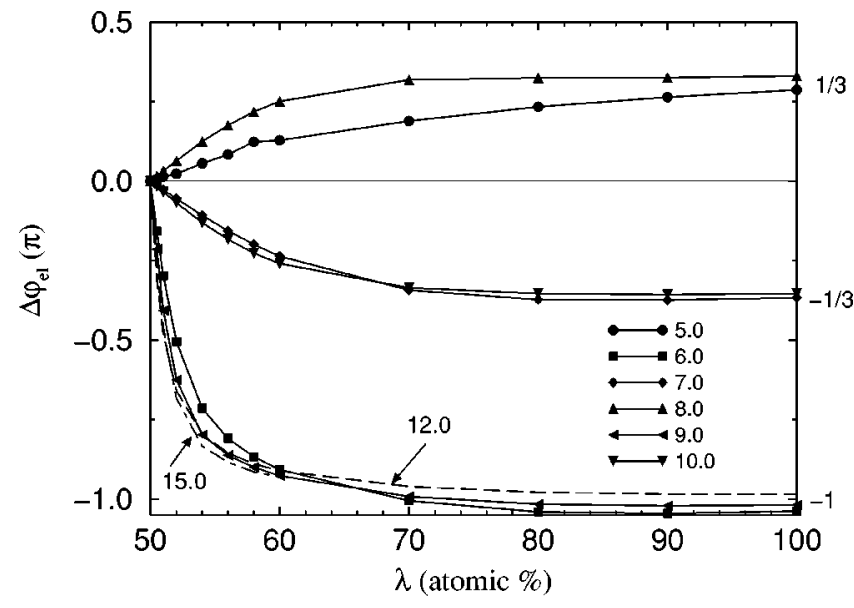

FIG. 2. Electronic-phase differences between the polar and nonpolar configurations for zigzag nanotubes.

corresponding wurtzite III-V and II-VI systems, ${ }^{19,20}$ where the spontaneous polarization can be viewed as the difference between the polarizations of the wurtzite (polar) and zincblende (nonpolar) geometries. Since these configurations become geometrically distinct only in the second shell of neighbors, their ionic phases are very close. The major contribution to the spontaneous polarization in wurtzite materials is then due to the difference between the electronic polarizations (which are $0.04-0.08 \mathrm{C} / \mathrm{m}^{2}$ ), while in BNNT's both ionic and electronic contributions are essential.

The ionic phase differences $\Delta \varphi_{i o n}$ between the polar and nonpolar configurations of zigzag nanotubes were evaluated via the virtual crystal approximation. The inset in Fig. 1 shows the results obtained by a simple lattice summation over the ionic charges [the first term in Eq. (3)], with the phases translated into the $[-\pi, \pi]$ interval. The phases plotted in the main graph were "unfolded" by eliminating all the $2 \pi$ discontinuities and setting the phase of the nonpolar reference configuration to zero. For the unfolded phases, as the diameter of a nanotube increases, i.e., as another hexagon is added around the circumference of the tube, the ionic phase goes up by $\pi / 3$, so that the total ionic phase for a $(n, 0) \mathrm{BN}$ nanotube amounts to $n \pi / 3$.

In Fig. 2 we show the electronic-phase differences $\Delta \varphi_{e l}$ between the polar and nonpolar configurations for zigzag nanotubes. These data suggest a natural division of the nanotubes into three families with different $\Delta \varphi_{e l}: \pi / 3$ for $n$ $=3 l-1,-\pi / 3$ for $n=3 l+1$, and $-\pi$ for $n=3 l$, where $l$ is an integer, ${ }^{21}$ which is similar to the result obtained by Mele and Král. ${ }^{7}$ However, the existence of such three classes of behavior is surprising, given that the ionic character of the electronic charge density (associated with the B-N bond) does not change with the nanotube index. Additionally, there is an important difference between our results and those of Ref. 7, where the electronic polarization of heteropolar nanotubes was studied within a simple $\pi$-orbital tight-binding approximation. In Ref. 7 , the " $n=3 l$ " family has a zero electronic phase instead of $-\pi$.

This discrepancy is due to the ambiguity of the definition of electronic polarization as a multivalued quantity, ${ }^{10}$ which can assume a lattice of values corresponding to Berry phases

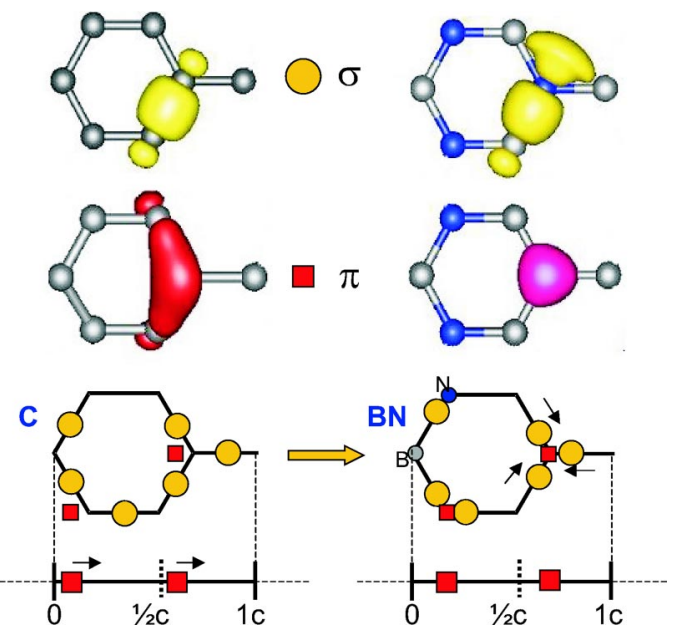

FIG. 3. (Color online) Upper panel: Examples of Wannier functions (WF) of the $\sigma$ and $\pi$ occupied bands of C (left panel) and BN (right panel) nanotubes. Lower panel: Schematic positions of the centers of the Wannier functions in $\mathrm{C}$ and $\mathrm{BN}$ hexagons, and the projections of the $\pi \mathrm{WF}$ onto the nanotube axes. The positions of the centers of $\sigma \mathrm{WF}$ are indicated by circles, and those of $\pi$ by squares. The direction of the shifts of $\sigma$ and $\pi \mathrm{WF}$ in an adiabatic transformation from $\mathrm{C}$ to $\mathrm{BN}$ is indicated by arrows. The projections of shifts of the $\sigma \mathrm{WF}$ cancel, so that the $\sigma \mathrm{WF}$ do not contribute to polarization (see text).

that differ by arbitrary multiples of $2 \pi$. Unlike the ionic phase model, where discontinuities in $\varphi_{i o n}(\lambda)$ can be easily monitored, Berry-phase calculations always produce phases that are smoothly folded into the $[-\pi, \pi]$ interval and cannot be extrapolated. To obtain an unambiguous determination of the spontaneous polarization of BNNT's of arbitrary diameters, one has to compute the polarization in a different way, using the centers of charge of the WF's of the occupied bands [Eq. (5)]. Note that this approach does not solve the problem of branch indetermination, since while Berry phases are defined modulo $2 \pi$, Wannier centers are defined modulo a lattice vector $\boldsymbol{R}$. However, by shifting the indetermination from the phase to the lattice vector, we are able to map the electronic polarization problem onto a simple electrostatic model, where the unfolding of the electronic phase is straightforward. ${ }^{10}$

\section{B. Maximally localized Wannier functions}

The results of the maximally localized WF calculations for BNNT's are summarized in Fig. 3, where examples of WF's for $\mathrm{C}$ and $\mathrm{BN}$ zigzag nanotubes of arbitrary diameter are shown, together with a schematic drawing that illustrates the shift of the Wannier centers in the adiabatic transformation from $\mathrm{C}$ to BN. Since

$$
\boldsymbol{P}_{e l}^{(B N)}=-\frac{2 e}{V} \sum_{i}\left(\boldsymbol{r}_{i}^{(B N)}-\boldsymbol{r}_{i}^{(C)}\right),
$$

the magnitude of the shift of the centers is directly proportional to the electronic polarization of the BNNT with respect to the nonpolar CNT. 
The $\sigma$-band WF's are centered in the middle of the C-C bonds in carbon nanotubes, while they are shifted towards the cations in BN nanotubes because of the different electronegativities of $\mathrm{B}$ and $\mathrm{N}$ atoms. Since these shifts have the same magnitude along each of the three bond directions, the vector sum of all shifts is zero (see bottom panel of Fig. 3), and the $\sigma$ orbitals do not contribute to the total polarization of the system. The $\pi$-band WF's are centered on the cations in BNNT's, while in CNT's they have a peculiar V shape, with centers somewhat outside of the C-C bond. The sum of the shifts of the $\pi$-band Wannier centers is nonzero only for the axial component, which means that the electronic polarization in BNNT's is purely axial.

The bottom panel of Fig. 3 shows the projection of the $\pi$ WF centers onto the axis of the tube. The projections of the centers have an effective periodicity of half of the axial lattice constant $c$, which leads to the indetermination of the electronic phase by multiples of $\pi$. Moreover, the WF description allows for an unambiguous unfolding of the electronic phase. In analogy to the ionic phase, we find that each individual hexagon carries a phase of $-\pi / 3$, leading to a total electronic phase of $-n \pi / 3$ for a $(n, 0)$ nanotube. This result demonstrates that the direction of the electronic polarization in a BNNT is specified by the orientation of the B-N bond and does not oscillate in direction with the nanotube diameter, contrary to the model Hamiltonian predictions. ${ }^{7}$ We should point out that the Wannier function results are completely consistent with the Berry-phase calculations, since an electronic phase of $-n \pi / 3$ for any $n$ can be folded, modulo $\pi$, into the three families found previously.

When we combine the results for the ionic and electronic phases into a general formula for the phase of an arbitrary $(n, m)$ BNNT,

$$
\Delta \Phi_{z}^{t o t}(n, m)=\Delta \varphi_{z}^{i o n}(n, m)+\Delta \varphi_{z}^{e l}(n, m)=\frac{n-m}{3}-\frac{n-m}{3},
$$

we find that the two contributions cancel exactly and the total spontaneous polarization in any BNNT is zero, i.e., the Wannier centers are arranged in such a way as to completely compensate the polarization due to ions. We have verified this result by two-point $(\lambda=50$ and $100 \%)$ calculations of the Berry-phase difference for a number of chiral nanotubes $[(3,1),(3,2),(4,1),(4,2),(5,2)$, and $(8,2)]$ and found an exact cancellation in all BNNT's, except for those narrower than $\approx 4 \AA$, where a residual polarization is present as an effect of the very high curvature. In such nanotubes Wannier centers cannot fully compensate the ionic polarization, due to the severe distortion of the atomic bonds, which makes these systems weakly pyroelectric. For example, $P=0.11 \mathrm{C} / \mathrm{m}^{2}$ in $(3,1), 0.008 \mathrm{C} / \mathrm{m}^{2}$ in $(7,0)$, and $0.002 \mathrm{C} / \mathrm{m}^{2}$ in $(12,0)$ nanotubes.

The exact cancellation is a result of the overall chiral symmetry of the nanotubes, which, although not centrosymmetric, are intrinsically nonpolar. Nevertheless, cancellation of ionic and electronic polarizations is exact only in the limit of an isolated BNNT. The spontaneous polarization in a nanotube bundle, where the chiral symmetry is effectively broken, is different from zero. For example, in $(7,0)$ bundles at equilibrium distance of $3.2 \AA P \approx 0.01 \mathrm{C} / \mathrm{m}^{2}$. However, in this case it is hard to estimate the separate contributions to polarization due to bundling, extreme curvature, and elastic deformation. Although smaller than in polymers or PZT, this polarization is comparable to some wurtzite pyroelectrics: e.g., $P=0.06 \mathrm{C} / \mathrm{m}^{2}$ in $w-\mathrm{ZnO}{ }^{20}$

\section{Piezoelectricity}

The Berry-phase method can also be employed to compute piezoelectric properties of BNNT's, which are directly related to polarization differences between strained and unstrained tubes. In the linear regime, the change in polarization due to strain can be decomposed into a sum of two terms: a uniform axial strain and a relative displacement of the two sublattices. It is therefore natural to describe the geometry of a BNNT of a given radius in terms of an axial lattice constant $c$ and an internal parameter $u$, where $u c$ is the length of the vector connecting the anion with the cation. With this choice, the axial piezoelectric polarization is

$$
\delta P_{3}=e_{33} \epsilon_{3}=\frac{\partial P_{3}}{\partial c}\left(c-c_{0}\right)+\frac{\partial P_{3}}{\partial u}\left(u-u_{0}\right),
$$

where the strain is $\epsilon_{3}=\left(c-c_{0}\right) / c_{0}$, and $c_{0}$ and $u_{0}$ are the equilibrium values of $c$ and $u$. The only surviving piezoelectric strain tensor component is

$$
e_{33}=e_{33}^{(0)}+\frac{e c_{0}^{2}}{V} N Z^{*} \frac{d u}{d c},
$$

where $N$ is the number of B-N pairs in the supercell. Here,

$$
e_{33}^{(0)}=c_{0} \partial P_{3} / \partial c
$$

is the "clamped-ion" piezoelectric constant (representing the effect of strain on the electronic structure), and

$$
Z^{*}=\left(V / e N c_{0}\right) \partial P_{3} / \partial u
$$

is the axial component of the Born dynamical charge tensor. Both polarization derivatives were computed as finite differences, changing $c$ or $u$ by $\pm 1 \%$. The parameter $\xi$ $=c_{0} d u / d c$, describing the change in the bond lengths under axial strain, was obtained by rescaling $c$ together with the associated components of ionic coordinates, and then relaxing the geometry of the system. For all the systems considered below, the value of $\xi$ is approximately the same and is equal to -0.085 .

We have calculated the piezoelectric properties for various bundles of zigzag BNNT's with individual diameters ranging from 3.9 to $10.2 \AA$. These results are summarized in Table I and compared to a few well-known piezoelectric and pyroelectric materials. While the piezoelectric constants of zigzag BNNT's are modest when compared with inorganic compounds, they are still substantially larger than those in the PVDF polymer family. 
TABLE I. Piezoelectric properties of zigzag BNNT bundles (Ref. 22). The corresponding values for a few piezoelectric materials are listed for comparison.

\begin{tabular}{lcccc}
\hline \hline$(n, m)$ & Diameter $(\AA)$ & $Z^{*}(\mathrm{e})$ & $\left|e_{33}\right|\left(\mathrm{C} / \mathrm{m}^{2}\right)$ & Ref. \\
\hline$(5,0)$ & 3.91 & 2.739 & 0.389 & \\
$(6,0)$ & 4.69 & 2.696 & 0.332 & \\
$(7,0)$ & 5.47 & 2.655 & 0.293 & \\
$(8,0)$ & 6.24 & 2.639 & 0.263 & \\
$(9,0)$ & 7.04 & 2.634 & 0.239 & \\
$(10,0)$ & 7.83 & 2.626 & 0.224 & \\
$(11,0)$ & 8.57 & 2.614 & 0.211 & \\
$(12,0)$ & 9.38 & 2.609 & 0.198 & \\
$(13,0)$ & 10.16 & 2.605 & 0.186 & \\
\hline$w-\mathrm{AlN}$ & & 2.653 & 1.50 & {$[19]$} \\
$w-\mathrm{ZnO}$ & & 2.11 & 0.89 & {$[20]$} \\
$\mathrm{PbTiO}$ & & & 3.23 & {$[2]$} \\
$\mathrm{P}(\mathrm{VDF} / \mathrm{TrFE})$ & & & $\approx 0.12$ & {$[3]$} \\
\hline \hline
\end{tabular}

\section{SUMMARY AND CONCLUSIONS}

In summary, we have investigated the spontaneous polarization and piezoelectric properties of $\mathrm{BN}$ nanotubes using state-of-the-art $a b$ initio methods. Our calculations demonstrate the complementary nature of Berry phase and Wannier function analysis, and show that a real-space description is necessary to unravel the Berry phases in complicated cases. The results suggest that BNNT's are excellent nonpolar piezoelectrics that exhibit substantially higher strain response than polar polymers. Moreover, we have shown that, contrary to the previous expectations, ideal noninteracting nanotubes are effectively nonpolar due to their intrinsic chiral symmetry, which leads to a total cancellation between the ionic and electronic polarizations. Breaking of this symmetry, as in the simple case of interacting nanotubes in a bundle, induces spontaneous polarization fields that are comparable to those of wurtzite semiconductors. Due to their piezoelectric and pyroelectric properties, BNNT's are excellent candidates for various nanoelectromechanical applications.

\section{ACKNOWLEDGMENTS}

We are indebted to N. Marzari and I. Souza for their invaluable help in computing Wannier functions in nanotubes. We would also like to thank D. Vanderbilt and R. Resta for illuminating discussions. This work was supported by NASA, ONR, and the Mathematical, Information and Computational Sciences Division, Office of Advanced Scientific Computing Reasearch of the U.S. D.O.E. under Contract No. DE-AC05-00OR22725 with UT-Battelle, LLC. The calculations were carried out at DoD and NC Supercomputing Centers.
*Electronic address: nakhmans@ @emo.physics.ncsu.edu

†Electronic address: mbnardelli@ncsu.edu

${ }^{1}$ T. Kumazawa, Y. Kumagai, H. Miura, M. Kitano, and K. Kushida, Appl. Phys. Lett. 72, 608 (1998); T. Yamamoto, Jpn. J. Appl. Phys., Part 1 37, 6041 (1998).

${ }^{2}$ G. Sághi-Szabó, R.E. Cohen, and H. Krakauer, Phys. Rev. Lett. 80, 4321 (1998); Phys. Rev. B 59, 12771 (1999).

${ }^{3}$ T. Furukawa, IEEE Trans. Electr. Insul. 24, 375 (1989); G. Eberle, H. Schmidt, and W. Eisenmenger, IEEE Trans. Dielectr. Electr. Insul. 3, 624 (1996).

${ }^{4}$ A. Rubio, J.L. Corkill, and M.L. Cohen, Phys. Rev. B 49, 5081 (1994); X. Blase, A. Rubio, S. G. Louie, and M. L. Cohen, Europhys. Lett. 28, 335 (1994).

${ }^{5}$ N.G. Chopra, R. J. Luyken, K. Cherrey, V. H. Crespi, M. L. Cohen, S. G. Louie, and A. Zettl, Science (Washington, DC, U.S.) 266, 966 (1995); A. Loiseau, F. Willaime, N. Demoncy, G. Hug, and H. Pascard, Phys. Rev. Lett. 76, 4737 (1996).

${ }^{6}$ P. Zhang and V.H. Crespi, Phys. Rev. B 62, 11050 (2000); H.F. Bettinger, T. Dumitrică, G. E. Scuseria, and B. I. Yakobson, ibid. 65, 041406 (2002).

${ }^{7}$ E.J. Mele and P. Král, Phys. Rev. Lett. 88, 056803 (2002).

${ }^{8}$ R.D. King-Smith and D. Vanderbilt, Phys. Rev. B 47, 1651 (1993); D. Vanderbilt, and R.D. King-Smith, ibid. 48, 4442 (1993).

${ }^{9}$ R. Resta, Rev. Mod. Phys. 66, 899 (1994).
${ }^{10}$ D. Vanderbilt, J. Phys. Chem. Solids 61, 147 (2000).

${ }^{11}$ N. Marzari and D. Vanderbilt, Phys. Rev. B 56, 12847 (1997); I. Souza, N. Marzari, and D. Vanderbilt, ibid. 65, 035109 (2002).

${ }^{12}$ E.L. Briggs, D.J. Sullivan, and J. Bernholc, Phys. Rev. B 54, 14362 (1996).

${ }^{13}$ D.M. Ceperley and B.J. Alder, Phys. Rev. Lett. 45, 566 (1980).

${ }^{14}$ J.P. Perdew and A. Zunger, Phys. Rev. B 23, 5048 (1981).

${ }^{15}$ N. Troullier and J.L. Martins, Phys. Rev. B 43, 1993 (1991).

${ }^{16}$ M. Fuchs and M. Scheffler, Comput. Phys. Commun. 119, 67 (1998).

${ }^{17}$ L. Kleinman and D.M. Bylander, Phys. Rev. Lett. 48, 1425 (1982).

${ }^{18}$ ABINIT is a joint project of the Université Catholique de Louvain, Corning Incorporated, and other contributors (URL: http:// www.abinit.org).

${ }^{19}$ A. Zoroddu, F. Bernardini, P. Ruggerone, and V. Fiorentini, Phys. Rev. B 64, 045208 (2001).

${ }^{20}$ F. Bernardini, V. Fiorentini, and D. Vanderbilt, Phys. Rev. B 56, R10 024 (1997); 63, 193201 (2001).

${ }^{21}$ In the limiting case of flat $\mathrm{C}$ or $\mathrm{BN}$ sheets, the electronic polarization is zero for any value of $\lambda$, due to the existence of a threefold symmetry axis perpendicular to the surface of the sheet.

${ }^{22}$ The data reported in Table I for nanotube bundles assume a closepacked geometry with intertube equilibrium distance of $3.2 \AA$. 